\title{
Driving impairs talking
}

\author{
Ensar Becic, Gary S. Dell, Kathryn Bock, and Susan M. Garnsey \\ University of Illinois at Urbana-Champaign, Urbana, Illinois
}

Tate Kubose

Exponent Engineering and Scientific Consulting, Menlo Park, California

AND

\author{
ARTHUR F. KRAMER \\ University of Illinois at Urbana-Champaign, Urbana, Illinois
}

\begin{abstract}
It is well known that conversation (e.g., on a cell phone) impairs driving. We demonstrate that the reverse is also true: Language production and comprehension, and the encoding of the products of comprehension into memory, are less accurate when one is driving. Ninety-six pairs of drivers and conversation partners engaged in a story-retelling task in a driving simulator. Half of the pairs were older adults. Each pair completed one dual-task block (driving during the retelling task) and two single-task control blocks. The results showed a decline in the accuracy of the drivers' storytelling and of their memory for stories that were told to them by their nondriving partners. Speech production suffered an additional cost when the difficulty of driving increased. Measures of driving performance suggested that the drivers gave priority to the driving task when they were conversing. As a result, their linguistic performance suffered.
\end{abstract}

Does driving an automobile hurt your ability to hold a conversation on a cell phone? We already know that talking on a cell phone makes driving more dangerous (Briem \& Hedman, 1995). We also know that conversing with a caller leads to slower responses to unexpected events (Strayer \& Drews, 2004), and that these costs are incurred regardless of whether the cell phone is hands free or handheld (Strayer \& Johnston, 2001). The impact of cell-phone conversation on driving has even been equated to that of drinking on driving (Strayer, Drews, \& Crouch, 2006). What we do not know is whether and how driving changes how, and how well, we talk.

We ask whether driving impacts speech production, comprehension, and the encoding of the products of comprehension into memory. To the layperson, conversing while driving seems easy. After all, driving uses the eyes, hands, and feet, whereas conversation requires only your mouth and ears. To the psychologist, these are multicomponent, albeit well-practiced, tasks that, despite employing different input and output channels, may share central resources. It turns out that little evidence is available on this question, because the many studies of conversation in automobiles have focused on driving, not on language. The two studies that did measure linguistic performance supported the lay intuition that language does not suffer from driving. Kubose et al. (2006) had drivers produce and comprehend statements about the locations of buildings in the town in which the study was conducted, and Tsimhoni, Green, and Lai (2001) tested the comprehension of spoken navigation, news, and e-mail messages. In both studies, accuracy was uncompromised by simulated driving compared with a parked-car control condition.

These null findings are puzzling. All accounts of how people produce language (e.g., Bock, 1982), understand it (e.g., Holmes \& Forster, 1970), and encode it into memory (e.g., Baddeley, 2003) have treated these processes as resource-demanding tasks. Talking and understanding, at least when the sentences express novel thoughts, are actually not easy. Furthermore, we know that drivers may prioritize driving - that is, devote resources to driving at the expense of other activities (Kramer, Cassavaugh, Horrey, Becic, \& Mayhugh, 2007). It follows that when driving is prioritized, language should suffer. So why do existing data say otherwise? It could be that such costs are small or that no study has provided a sensitive enough measure. Or it could be that no study has made the simulated driving a sufficient priority. In the present study, we investigated the possible costs to language from driving under different circumstances (e.g., intersection crossing vs. routine driving) and within different subject groups (older vs. younger adults) that would be expected to affect the extent to which driving is prioritized.

G. S. Dell, gdell@cyrus.psych.uiuc.edu 
The present study assessed the effects of driving on speech production, comprehension, and memory. To accomplish this goal, we used a story-retelling task in which participants heard and then retold short narratives, each of which described a single event (e.g., a robbery or a trip; Brown \& Dell, 1987; Lockridge \& Brennan, 2002). Although this approach does not create a freewheeling natural conversation, describing an event in a $10-30$-sec retelling is, we claim, a common component of conversation. More importantly, this method affords control, enforces the use of novel sentences, and ensures a certain level of engagement in the conversation. We were thus able to assess the accuracy of the speech production and later memory for the material. We also compared the effects of routine driving and of more attention-demanding driving on performance with the story. If dual-task costs are exhibited in a speech task, we would expect the cost to be particularly exacerbated in driving situations that require greater resources from the driver.

In addition, we examined whether the impact of driving on speech processing differs between older and younger drivers. Although the deficits that older adults exhibit in dual tasks have been well established (Salthouse, 1996), these individuals also have much greater driving experience, which may reduce dual-task penalties (Kramer et al., 2007; Strayer \& Drews, 2004). Older adults also tend to emphasize accuracy over speed in performing a variety of tasks (Band \& Kok, 2000). Consequently, we tested whether older drivers exhibited a relatively greater level of prioritization of driving and whether this then had a greater impact on their performance with the stories.

Finally, we also assessed the often overlooked impact of language use on routine driving - that is, on driving in situations that do not require speeded responding. Kubose et al. (2006) found that, during routine driving, speech processing increases the variability in velocity control but decreases variability in maintenance of lane position, suggesting that the impact of language use on continuous measures of routine driving is not straightforward. We emphasize again, however, that our focus was chiefly on the effects of driving on language, rather than the other way around.

\section{METHOD}

\section{Participants}

Ninety-six pairs of adults participated in the study. Each pair consisted of a driver and a passenger or caller. Half of the participant pairs were older adults $(M=70.7$ years, $S D=7.05)$ and half were younger adults $(M=19.6$ years, $S D=1.4)$. One participant in each pair was assigned the role of the driver, and the other was designated as either the passenger, who sat next to the driver in the simulator, or the caller, who occupied a soundproof booth that was outside of the simulator. These roles, which were counterbalanced across gender, did not change during the experiment. All participants were administered questionnaires about their driving habits (i.e., accident rate, miles driven, years of driving experience), which revealed no unanticipated age-related differences. Older participants were highfunctioning, community-dwelling individuals who drove regularly.

\section{Apparatus and Materials}

Driving simulator. This study was conducted in the Beckman Driving Simulator at the University of Illinois (GlobalSim, Inc. Vec- tion Research Simulator). This is a fixed-base simulator consisting of a 1998 Saturn SL with a $135^{\circ}$ wraparound projection screen. All participants were equipped with headphones and microphones.

Speech task. Participants listened to and then retold short narratives to their partner. Sixteen such stories were selected from Lockridge and Brennan (2002), and they were read aloud and recorded for presentation to participants. The average story was $12.7 \mathrm{sec}$ long.

Memory test. After each block in which participants performed the speech task (two such blocks), they were asked to leave the simulator and to recall everything that they remembered about the stories that were told to them by their partner.

\section{Procedure}

Drivers and their partners first completed a practice drive to familiarize themselves with the simulator and then practiced the speech task with the car parked. Finally, they completed three critical task blocks consisting of two single-task blocks (a driving-only and a speech-only block) and one dual-task block (driving while conversing). Block order was counterbalanced. In the dual-task block, of course, only the driver received dual-task treatment (driving while performing the speech task). The passenger or caller participated in only the speech-task portion of the dual-task block. During the speech-only block, the driver and passenger were in the car, but it was parked.

The driving task consisted of driving through an urban environment and crossing several busy intersections. Drivers were instructed to obey the $30 \mathrm{mph}$ speed limit, stay in the center of their lane, stop at stop signs, and cross the intersections in a safe and timely manner.

In the speech task, participants heard stories through their headphones that were not presented to their partners. They then retold the stories in their own words to their partner, under instructions to tell the story in a way that would promote good recollection by their partner. After hearing each story, the reteller had $30 \mathrm{sec}$ to tell it. At the end of each story-retelling segment, another prompt announced that the other member of the pair was about to hear a story, and the procedure was repeated, with the roles of reteller and listener switched. Each member of the pair heard and then retold four stories during the two blocks in which participants performed the speech task. The assignment of stories to blocks and conditions was counterbalanced.

Participants were instructed to refrain from talking to each other during the study, except when they were performing the speech task.

\section{RESULTS}

\section{Driving Performance}

Driving performance was investigated through continuous as well as discrete driving measures. Continuous measures focused on straightaway sections of the route (from $100 \mathrm{~m}$ after one intersection to $100 \mathrm{~m}$ before the next intersection), whereas discrete measures analyzed the driver's handling of intersections. Both kinds of measures were analyzed in a $2 \times 2 \times 2$ mixed-design matrix with task (single and dual) as a within-subjects measure and with age and location of conversation partner (inside vehicle and outside vehicle) as between-subjects measures.

Continuous-driving measures. Continuous-driving performance was assessed by the mean and standard deviation of velocity (meters per second) and lane keeping (meters from center lane). There was a significant effect of task on the variability of lane keeping $[F(1,92)=222.9$, $p<.001]$ and on the variability of velocity $[F(1,92)=$ $13.62, p<.001]$. Drivers exhibited greater variability in both lane keeping and velocity when driving only compared with when they performed the speech task while 
Table 1

Main Effects of Continuous and Discrete Measures on Driving Performance

\begin{tabular}{|c|c|c|c|c|c|c|}
\hline & \multicolumn{4}{|c|}{ Continuous Measures } & \multicolumn{2}{|c|}{ Discrete Measures } \\
\hline & \multicolumn{2}{|c|}{ Lane Position (m) } & \multicolumn{2}{|c|}{ Velocity $(\mathrm{m} / \mathrm{sec})$} & \multirow{2}{*}{$\begin{array}{c}\text { Braking } \\
\text { Distance }(\mathrm{m})\end{array}$} & \multirow{2}{*}{$\begin{array}{c}\text { Time to } \\
\text { Cross (sec) }\end{array}$} \\
\hline & $M$ & $S D$ & $M$ & $S D$ & & \\
\hline \multicolumn{7}{|l|}{ Task } \\
\hline Single & .25 & .16 & 12.7 & .90 & $\underset{* *}{49.0}$ & 42.8 \\
\hline Dual & .22 & .10 & 12.2 & .74 & 43.7 & 43.9 \\
\hline \multicolumn{7}{|l|}{ Age } \\
\hline Younger & .28 & .13 & $\underset{*}{12.8}$ & .67 & 41.7 & $\underset{* * *}{26.8}$ \\
\hline Older & .20 & .13 & 12.1 & .97 & 50.0 & 59.9 \\
\hline \multicolumn{7}{|l|}{ Location } \\
\hline Inside & .24 & .13 & 12.5 & .77 & 48.0 & 41.7 \\
\hline Outside & .23 & .12 & 12.4 & .87 & 44.6 & 45.1 \\
\hline
\end{tabular}

driving (see Table 1 and Figure 1). The analysis of mean velocity $[F(1,92)=23.23, p<.001]$ showed that participants drove more slowly when conversing compared with when they performed only the driving task.

Velocity was the only measure of variability that showed a significant main effect of age $[F(1,92)=16.57, p<$ $.001]$, with older drivers exhibiting greater variability in velocity than did younger drivers (see Table 1). The analysis of variability in velocity also revealed a significant interaction between age and task condition $[F(1,92)=$ $18.38, p<.001]$. Older drivers' velocity was less variable when conversing $(M=0.8 \mathrm{~m} / \mathrm{sec})$ than when they performed the driving task only $(M=1.14 \mathrm{~m} / \mathrm{sec})[F(1,47)=$ $21.98, p<.001]$, whereas task load had no such effect for younger drivers $(p>.56)$. As expected, the analysis of mean velocity revealed that older drivers drove more slowly $[F(1,92)=4.05, p=.047]$, providing evidence of older drivers' defensive driving.

\section{Variability in Lane Keeping}

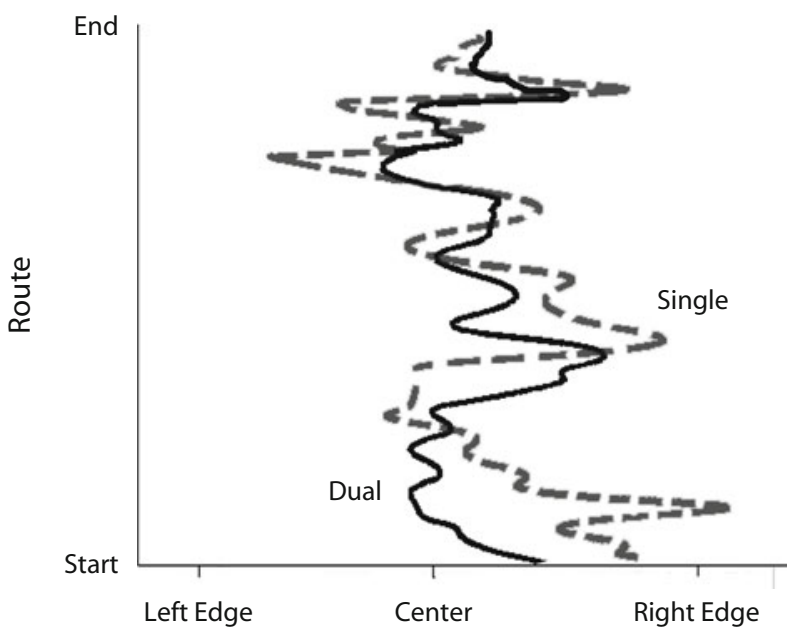

Lane Position

Figure 1. Graphic portrayal of variability in lane keeping for a randomly selected participant as a function of task condition.
The continuous-driving measure analyses yielded no significant effects involving the location of the partner.

Discrete driving measures. Drivers' handling of intersections was assessed through two discrete variables: braking distance from the intersection stop sign, and time to cross the intersection. The analysis of braking distance revealed that drivers braked closer to the stop sign when engaged in the speech task than when driving in silence [see Table $1 ; F(1,92)=9.89, p=.02$ ]. This result remained significant even when velocity (at $100 \mathrm{~m}$ before the stop sign) was included as a covariate $[F(1,91)=6.41$, $p=.013]$. The analysis also demonstrated that older drivers braked farther away from the intersection than did younger drivers $[F(1,91)=15.4, p<.001]$, consistent with older drivers' tendency toward more cautious driving behavior

As Table 1 shows, older drivers required much more time to cross intersections $[F(1,92)=55.8, p<.001]$, but this age effect did not interact with task. The older adults drove defensively in this respect, regardless of whether they were engaged in the speech task.

Location of the conversation partner did not affect discrete driving measures.

\section{Speech-Task Performance}

The performance on the speech task was evaluated in two ways. Accuracy of immediate story retelling explored the impact of driving on comprehension, verbal working memory, and production. The later recall test evaluated both the level of engagement in comprehending the partner's speech and the quality of long-term encoding of the same. Unless noted, all analyses were performed as a fourway mixed-mode ANOVA, with task as a within-subjects factor and with age, location of the conversation partner, and participant's role (driver and nondriver) as betweensubjects factors.

Accuracy of story retelling. The accuracy of story retelling was measured as the proportion of propositions reported correctly over the total number of propositions, giving us a gist scoring measure. As shown in Table 2 and Figure 2, participants exhibited more accurate story retelling when not driving $[F(1,182)=75.9, p<.001]$. As ex- 
Table 2

Main Effects of Accuracy of Retelling and Recall

\begin{tabular}{ccc}
\hline & $\begin{array}{c}\text { Accuracy } \\
\text { of Retelling }\end{array}$ & $\begin{array}{c}\text { Accuracy } \\
\text { of Recall }\end{array}$ \\
\hline Task & .68 & .54 \\
Single & ${ }^{* * *}$ & $* * *$ \\
Dual & .62 & .47 \\
Age & .72 & .67 \\
Younger & ${ }^{* * *}$ & $* * *$ \\
Older & .59 & .34 \\
Location & .66 & .53 \\
Inside & .65 & .48 \\
Outside & .64 & .46 \\
Role & $*$ & .55 \\
Driver & .67 & \\
Nondriver & &
\end{tabular}

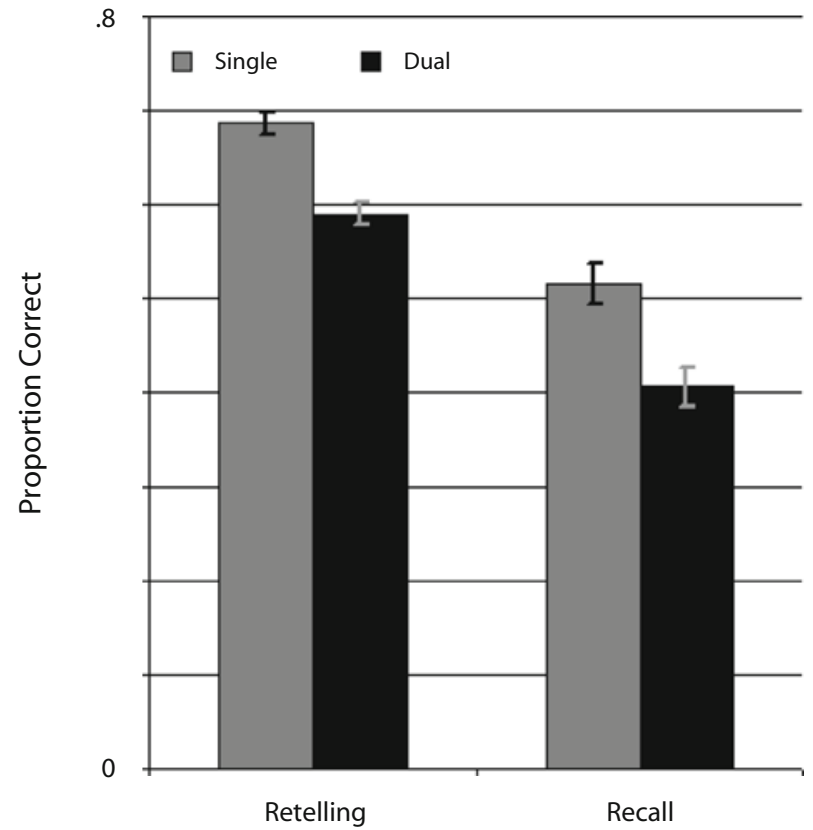

Figure 2. Proportion of accurately retold and recalled propositions as a function of task condition only for drivers. The bars represent standard errors.

pected, younger participants were more accurate in story retelling than were their older counterparts $[F(1,182)=$ $79.87, p<.001]$. Also, the analysis revealed a significant main effect of participant role $[F(1,182)=4.57, p=.034]$ and a task $\times$ role interaction $[F(1,182)=33.38, p<.001]$. The accuracy of retelling performance was equivalent between driver and nondriver when they performed only the speech task $(p>.7)$. When driving, though, the drivers displayed poorer accuracy of retelling $(M=.59)$ than did nondrivers $(M=.66)[F(1,188)=13.34, p<.001]$.
If it is assumed that driving through an intersection requires additional attentional resources, then linguistic performance should suffer to the extent that it calls on those resources. In our analyses per roadway section, we compared the accuracy of retelling of stories that participants both heard and retold within the same route section (intersection and straightaway). Since this is a measure of accuracy in the dual-task condition only, the analysis was carried out within the four-way ANOVA, with age, location, and role as between-subjects measures and with route (intersection, straightaway) as a within-subjects measure. The analysis uncovered a significant role $\times$ route interaction $[F(1,72)=$ $5.42, p=.023]$, illustrated in the top of Figure 3. Route affected the drivers $[F(1,37)=4.39, p=.043, M=.61$ for straightaways, and $M=.52$ for intersections] but not the nondrivers $(p>.27)$. Drivers were less accurate in their retellings when experiencing a more demanding driving situation, providing further evidence of prioritization and a knock-on cost to linguistic processing.

In summary, consistency in driving performance while dealing with speech (less driving variability; see Table 1) came at the expense of accuracy in story retelling. When

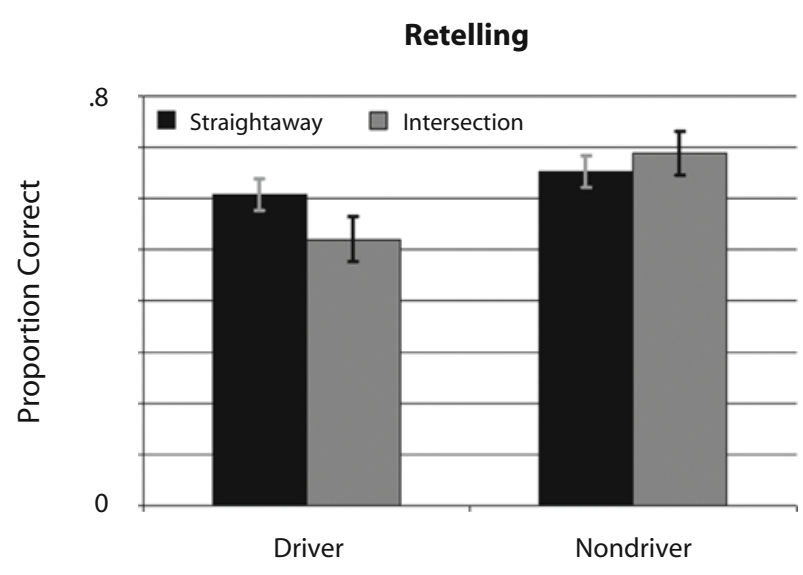

Recall

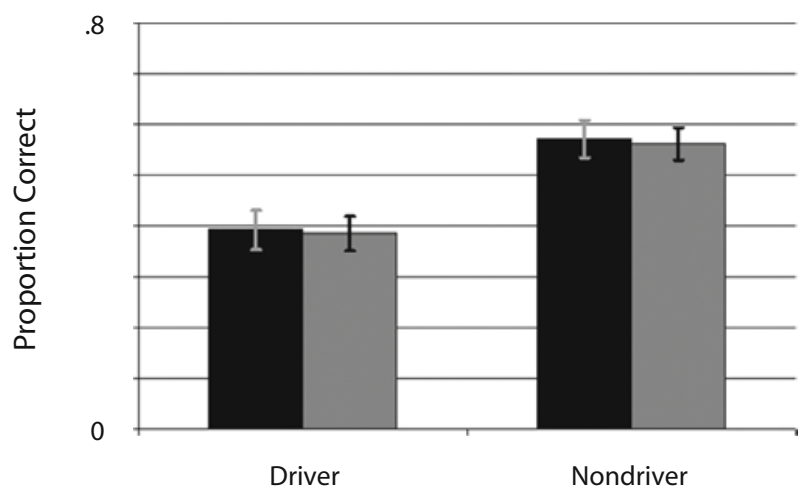

Figure 3. The top panel represents the proportion of accurately retold stories, and the bottom panel represents the proportion of accurately recalled story propositions as a function of participant's role and section of the route. The bars represent standard errors. 
doing the speech task only, drivers and nondrivers were equally good at retelling. When the car was moving, however, drivers displayed a large decline in speech-task performance.

Memory test performance. The final recall of all stories represents the ultimate evaluation of success in the speech task. Given the variability in accuracy of retelling, some participants were presented with fewer details to remember than were others. To assess the amount of recall independently of this variability, the percentage of propositions that were recalled (per story) was divided by the percentage of propositions that were accurately retold to participants by their partner, giving a measure of proportional gist scoring.

As Table 2 shows, there was better recall of stories by the proportional gist measure when the participants were performing only the speech task during encoding, compared with when one of them was driving $[F(1,182)=$ $15.82, p<.001]$. As expected, older participants exhibited poorer recall of stories than did their younger counterparts $[F(1,182)=195.92, p<.001]$. Also, participants in the inside-vehicle condition showed better recall than did those in the outside-vehicle condition $[F(1,182)=5.12$, $p=.025]$, demonstrating a small but reliable cost to communication among distant participants.

The analysis of proportional gist scoring also revealed a main effect of role $[F(1,182)=14.89, p<.001]$ and a significant task $\times$ role interaction $[F(1,182)=4.38, p=$ .038]. Drivers exhibited poorer recall when stories were presented under dual-task conditions $(M=.41)$ compared with when they performed only the speech task $(M=.51)$ $[F(1,94)=15.53, p>.001]$, whereas the nondrivers did not show a decline in recall as a function of task load $(p>.14)$.

As we did for the accuracy of story retelling, we compared recall for stories that participants heard on a straightaway with recall for those that they heard on an intersection portion of the route. The same four-way mixed-mode ANOVA was performed on the proportional gist scoring measure of recall, yielding no significant effects of route $(p>.82)$ or role $\times$ route interaction $(p>.97)$. Drivers (as well as nondrivers) exhibited equivalent recall for stories irrespective of the route section on which they were heard (see bottom of Figure 3). Unlike accuracy of story retelling, the difficulty of route had no effect on recall performance.

\section{Individual Differences in \\ Driving-Speech Interaction}

In order to test the idea that driving is prioritized in dual-task situations, we also assessed the extent to which individual differences in driving and speech were linked. Recall that velocity became less variable when the driving occurred during the speech task (Table 1). It turns out that the amount of this change was somewhat greater for drivers who did worse on the speech task. The correlation between the change in velocity variability (from single task to dual task) and recall (dual task only) was significant $(r=-.23, p=.025)$, and the correlation between change in velocity variability and retelling accuracy was nearly so $(r=-.20, p=.053)$. Poorer language users thus changed their driving more when using language. No doubt some of these individual differences are associated with age. As reported above, older drivers' velocity was less variable under dual-task conditions than when driving silently, but this difference was not seen in younger drivers. Moreover, older drivers performed worse overall on the speech tasks than did younger ones. The drivers who performed more poorly in language use (more likely to be older) were thus the ones who reduced their velocity variability more when using language. Older individuals may implicitly believe that using language requires more effort for them and that their driving abilities may therefore suffer when they are conversing. Consequently, they protect their drivingmaking it less variable-when doing the speech task.

\section{DISCUSSION}

We asked whether driving an automobile interferes with the ability to process and remember language. The answer is unequivocally affirmative. Driving negatively impacts story retelling as well as the process of comprehending and encoding stories into long-term memory. More stable driving (lower variability in velocity and lane keeping) while using language came at the expense of accuracy in speech-task performance (see Figure 1). These results indicate that driving took priority over conversation.

The prioritization of driving was also seen in the influence of route difficulty. When crossing intersections - a part of the route that requires more attentional resourcesdrivers, but not nondrivers, exhibited an additional cost in the accuracy of their retellings compared with their performance when driving on a less demanding straightaway part of the route (see Figure 2). Final recall of the stories was likewise negatively affected by the dual task.

Driving-route difficulty (intersection vs. straightaway) did not affect recall, in contrast to its effect on story retelling. One explanation for this disparity is in the differences between comprehension and production. Drivers who were retelling stories were involved in a dual task during both the comprehension and the production portion of the retelling task. However, later story recall (which was performed as a single task) isolates the effect of the dual-task load during the earlier comprehension and encoding of stories. This result allows us to make some inferences regarding the origin of the cost to driving in comprehension versus production. Since final recall-which depends on the comprehension and encoding of stories - was not affected by route difficulty, we can conclude that the more demanding intersection condition imposed more of a cost on speech production than did comprehension. That is, there is a general dual-task cost to comprehension and encoding that is independent of driving difficulty, and an extra cost in production quality that was associated with the intersection. Of course, it is quite possible that excessively difficult driving (e.g., driving in a blizzard) would further degrade comprehension as well.

Our conjecture that more demanding driving produces greater costs thus proved to be correct. Speech processing, 
however, deteriorated even during performance of routine driving: Driving on straightaways led to poorer processing and memory for speech that was heard during these easy route segments (Figure 3 ). It is noteworthy that the poor linguistic performance during both demanding and routine driving was true specifically for the driver rather than for the conversation partner. This fact allows us to conclude that the difficulty is not created by factors that impact the partner and driver equally, such as the limited opportunity for eye contact between the participants when someone is driving.

How do we account for our finding that routine driving is actually more stable (less variable in lane keeping and velocity) during conversation? First, we note that this counterintuitive finding matches that of Kubose et al. (2006) with respect to variability in lane position. Although conversation clearly acts as a distraction in situations in which a fast response is required (e.g., avoiding a vehicle that ran a red light; Strayer \& Drews, 2004), it is possible that conversation actually aids in the performance of highly routinized tasks, such as driving, when nothing unexpected happens. Taking attention away from performance has been found to be advantageous for highly practiced and automatized psychomotor skills (see, e.g., Beilock, Afremow, Rabe, \& Carr, 2001). A strategic compensatory response to additional task demands offers an alternative explanation. In largely uneventful traffic scenarios such as ours, the cost of changes in velocity or of weaving within one's lane is small, so the incentive for more stable driving is also small. Conversation may act as such an incentive (e.g., a more demanding driving situation), increasing drivers' attentiveness and thereby improving their performance (see Laberge, Scialfa, White, \& Caird, 2004, with respect to variability in lane position). It remains to be explored whether conversation has the same advantage when driving requires constant attentional updating along a difficult route (e.g., driving across a snowy mountain pass). Finally, we should consider the possibility that the reduced variability in lane position and velocity that is associated with language use is actually not a good thing. A variably moving system is potentially a more responsive system; thus, the greater variability in the continuous driving measures when there is no conversation may help explain nonconversing drivers' faster reactions to sudden external events. In any event, it is clear from our research that, regardless of whether conversation is detrimental to driving under the conditions that we tested, the cost of performing a dual task exists; it exists in the quality of the linguistic performance.

As expected, older drivers exhibited more cautious driving practices, such as taking longer to cross intersections; and, although they showed greater variability in velocity than did younger drivers, unlike the younger drivers, they significantly reduced this variability when doing the speech task. Their poorer performance on the speech task was the result of expected consequences of age-related declines in working and short-term memory (Bopp \& Verhaeghen, 2005). Older drivers did not exhibit a greater dual-task cost in linguistic performance than younger drivers, however. That is, age and task did not interact on measures of retelling and recall. Greater driving (and talking) experience may provide older drivers with enough reserve to ameliorate the general dual-task decline that is associated with aging (Pashler \& Johnston, 1998) as well as enable aging drivers to avoid potential collisions (Kramer et al., 2007).

To summarize, much of the research on driving and cell-phone use has emphasized the costs of speech production and comprehension on driving. We turned this emphasis around. Although intuition suggests that language is little affected by routine driving, this intuition is incorrect. Speaking, comprehending, and remembering are demanding tasks. After all, most sentences that we hear and say are novel, a fact of human language that was brought to the attention of psychologists by Chomsky's (1959) review of Skinner's Verbal Behavior. Understanding a story that you have never experienced before and producing a comprehensible retelling of it to a partner who must remember it requires the construction of representations of novel combinations of cognitive objects at multiple processing levels. Central processing resources are clearly required (see, e.g., Bock, 1982). Thus, if motorists are rational and prioritize their driving - as we and others (e.g., Kramer et al., 2007) have shown - it follows that other demanding concurrent tasks, such as language use, should experience costs. This analysis finds support in our data.

We conclude with a practical implication. Studies that are relevant to legislation regarding the use of cell phones while driving are naturally concerned with costs and benefits. Our research shows that such studies should not presume that dual-task costs (if any) are absorbed solely by driving. Our data clearly show that language use, under the conditions of our experiment, is degraded by even the most routine driving. Given this finding, important conversations (e.g., business negotiations) might become a matter of extra concern. Either the conversation suffers because driving remains protected, or, worse, the priority shifts to the conversation, and the driving suffers - catastrophically.

\section{AUTHOR NOTE}

We thank the National Institute on Aging (Grants RO1 AG25667 and RO1 AG25032) and General Motors for the support of our research. We also thank Matt VanPatter for his assistance with the experiment. Correspondence concerning this article should be addressed to G. S. Dell, Beckman Institute, University of Illinois, 405 N. Mathews Ave., Urbana, IL61801 (e-mail: gdell@cyrus.psych.uiuc.edu).

\section{REFERENCES}

BADDELEY, A. (2003). Working memory: Looking back and looking forward. Nature Reviews Neuroscience, 4, 829-839. doi:10.1038/ nrn1201

Band, G. P. H., \& KoK, A. (2000). Age effects on response monitoring in a mental-rotation task. Biological Psychology, 51, 201-221. doi:10.1016/S0301-0511(99)00038-1

Beilock, S. L., Afremow, J. A., Rabe, A. L., \& Carr, T. H. (2001). "Don't miss!" The debilitating effects of suppressive imagery on golf putting performance. Journal of Sport \& Exercise Psychology, 23, 200-221. 
Bock, J. K. (1982). Toward a cognitive psychology of syntax: Information processing contributions to sentence formulation. Psychological Review, 89, 1-47. doi:10.1037/0033-295X.89.1.1

Bopp, K. L., \& Verhaeghen, P. (2005). Aging and verbal memory span: A meta-analysis. Journal of Gerontology, 60B, 223-233.

Briem, V., \& Hedman, L. R. (1995). Behavioural effects of mobile telephone use during simulated driving. Ergonomics, 38, 2536-2562. doi:10.1080/00140139508925285

Brown, P. M., \& Dell, G. S. (1987). Adapting production to comprehension: The explicit mention of instruments. Cognitive Psychology, 19, 441-472. doi:10.1016/0010-0285(87)90015-6

Chomsky, N. (1959). A review of B. F. Skinner's Verbal Behavior. Language, 35, 26-58.

Holmes, V. M., \& Forster, K. I. (1970). Detection of extraneous signals during sentence recognition. Perception \& Psychophysics, 7, 297 301 .

Kramer, A. F., Cassavaugh, N., Horrey, W. J., Becic, E., \& MayHUGH, J. L. (2007). Influence of age and proximity warning devices on collision avoidance in simulated driving. Human Factors, 49, 935 949. doi:10.1518/001872007X230271

Kubose, T. T., Bock, K., Dell, G. S., Garnsey, S. M., Kramer, A. F., \& Mayhugh, J. [L.] (2006). The effects of speech production and speech comprehension on simulated driving performance. Applied Cognitive Psychology, 20, 43-63. doi:10.1002/acp.1164

Laberge, J., Scialfa, C., White, C., \& CAird, J. (2004). Effects of passenger and cellular phone conversations on driver distraction. Transportation Research Record, 1899, 109-116. doi:10.3141/1899-15
Lockridge, C. B., \& Brennan, S. E. (2002). Addressees' needs influence speakers' early syntactic choices. Psychonomic Bulletin \& Review, 9, 550-557.

Pashler, H., \& Johnston, J. C. (1998). Attentional limitations in dualtask performance. In H. Pashler (Ed.), Attention (pp. 155-189). East Sussex, U.K.: Psychology Press.

Salthouse, T. A. (1996). The processing-speed theory of adult age differences in cognition. Psychological Review, 103, 403-428. doi:10.1037/0033-295X.103.3.403

Strayer, D. L., \& Drews, F. A. (2004). Profiles in driver distraction: Effects of cell phone conversations on younger and older drivers. Human Factors, 46, 640-649. doi:10.1518/hfes.46.4.640.56806

Strayer, D. L., Drews, F. A., \& Crouch, D. J. (2006). A comparison of the cell phone driver and the drunk driver. Human Factors, 48, 381 391. doi:10.1518/001872006777724471

Strayer, D. L., \& Johnston, W. A. (2001). Driven to distraction: Dual-task studies of simulated driving and conversing on a cellular telephone. Psychological Science, 12, 462-466. doi:10.1111/1467 $-9280.00386$

Tsimhoni, O., Green, P., \& LAi, J. (2001). Listening to natural and synthesized speech while driving: Effects on user performance. International Journal of Speech Technology, 4, 155-169. doi:10.1023/ A:1011387612112

(Manuscript received May 24, 2009; revision accepted for publication October 14, 2009.) 